\title{
On the Practical Implementation of Feedforward Control Signals Given in Polynomial Form
}

\author{
Paola Gervasio \\ Dipartimento di Matematica \\ University of Brescia \\ Brescia, Italy \\ paola.gervasio@ing.unibs.it
}

\author{
Stefano Piccagli, Antonio Visioli \\ Dipartimento di Elettronica per l'Automazione \\ University of Brescia \\ Brescia, Italy \\ \{stefano.piccagli,antonio.visioli\}@ing.unibs.it
}

\begin{abstract}
A Chebyshev optimization approach can be employed effectively for the determination of the (reference) command input to be applied to a feedback PID control system in order to achieve a minimum-time output transition subject to constraints on both the control variable and the system output. The resulting command input function is given in polynomial form, for which a practical implementation can be difficult. In this paper we propose a method for the determination of a stable low-order filter whose step response approximates the open-loop command signal so that a standard two degree-of-freedom controller results. Simulation examples show that the technique is effective and yields a stable transfer function which can be easily implemented into a DCS control system.
\end{abstract}

\section{Introduction}

It is well-known that Proportional-Integral-Derivative (PID) controllers are the most widely adopted controllers in industry owing to the advantageous cost/benefit ratio they are able to provide. In order to help the operator to select the controller gains to address given control specifications, many tuning formulas have been devised in the past [9] and autotuning functionalities are almost always available in commercial products $[2,7]$.

In any case, the typical problem that has to be tackled in finding the controller parameters is addressing the setpoint following and the load disturbance rejection performance at the same time. In this context, the use of a two degree-of-freedom controller is an effective solution and the use of the well-known set-point weighting strategy [1] falls in this framework. In general, the two degree-offreedom control strategy consists in tuning the feedback PID controller in order to achieve a satisfactory load disturbance rejection performance and then implementing a feedforward controller to recover the set-point following performance. In this context, many methodologies have been devised for the design of the feedforward controller
$[5,6,14]$. In particular, a methodology for the determination of minimum-time feedforward control to be applied to a PID control loop where both actuator limits as well as constraints on the maximum overshoot and undershoot are taken into account has been proposed in [11]. In other words, the command input to be applied to the closed-loop system is determined in order to provide a minimum-time rest-to-rest transition from an equilibrium state to another (corresponding to a process output transition from a setpoint value to another) subject to minimum and maximum constraints for the manipulated variable as well as for the process output.

A Chebyshev approach is employed for this purpose [4][15], namely, the state variables and the control variable are parameterized by Chebyshev series. In this way the system dynamics is transformed into a system of algebraic equations and therefore the minimum-time control problem is reduced into a constrained optimization problem. However, the determined command signal is in polynomial form and therefore its implementation in a practical context, namely, by means of a Distributed Control Systems (DCS) can be difficult. Thus, we propose a technique for the design of a (stable and low-order) filter whose response to a step signal approximates a polynomial input command and can therefore be implemented easily with standard DCS blocks (for example, lead/lag or lag blocks). In particular, the input function is rewritten into a monomial form and it is compared by the monomial generated by the Chebyshev approximation of the Laplace inverse transform of a rational stable transfer function with a given number of real poles and zeros.

It is worth noting that applying the standard inverse Laplace transform operator to the polynomial function obtained from the Chebyshev optimization is not a viable solution because the resulting filter is a high-order integrator and it is therefore unstable (this fact also prevents from the application of model-order reduction techniques such as, for example, balanced truncation (see [3, 13]).

The paper is organised as follows. In Section 2 we review the Chebyshev approach for the determination of the command function to be applied to the closed-loop sys- 


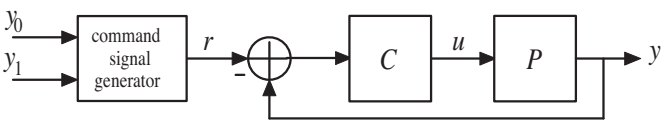

Figure 1. The control scheme for the Chebyshev approach.

tem in order to minimise the output transition time subject to constraints both on the manipulated and on the process variable. In Section 3 we present the methodology for the determination of the filter whose step response approximates the command input. Practical issues are addressed in Section 4. Illustrative examples are shown in Section 5 whereas conclusions are drawn in Section 6.

\section{Chebyshev technique for minimum-time process variable transition}

In this section we briefly review the Chebyshev approach for the determination of the command signal $r(t)$ that provides a minimum-time process variable transition subject to constraints on both the manipulated and the process variable. Details can be found in [11].

\subsection{Problem formulation}

We consider the unity-feedback control loop shown in Fig.1, where the process to be controlled (assumed to be self-regulating) is modelled as a first-order-plus-deadtime (FOPDT) transfer function

$$
P(s)=\frac{K}{T s+1} e^{-L s}
$$

where the dead time is approximated by a first-order Padè approximation, i.e. we consider:

$$
\tilde{P}(s)=\frac{K}{T s+1} \frac{-\frac{L}{2} s+1}{\frac{L}{2} s+1} .
$$

This is a typical choice in industrial practice, since this model can describe well the dynamics of many industrial processes. The feedback controller is of (output-filtered) PID type, whose transfer function is:

$$
C(s)=K_{p}\left(1+\frac{1}{T_{i} s}+T_{d} s\right) \frac{1}{T_{f} s+1}
$$

where $K_{p}$ is the proportional gain, $T_{i}$ is the integral time constant, $T_{d}$ is the derivative time constant and $T_{f}$ is the time constant of a first-order filter that makes the transfer function proper. The value of $T_{f}$ can be selected, once the other parameters are determined, such that the filter dynamics does not influence the dynamics of the PID controller and the effects of the measurement noise are reduced as much as possible.
An approach based on Chebyshev polynomials can be employed for a command signal generator in order to determine the signal $r(t)$, to be applied to the closed-loop system when a transition from an equilibrium point corresponding to a process output value $y_{0}$ to another equilibrium point corresponding to a process output value $y_{1}$ is required. In particular, a minimum-time (rest-to-rest) transition is required subject to given limits on the control variable and on the process variable. In the following, without loss of generality, we consider $y_{0}=0$ and $y_{1}=1$. The considered time-optimal feedforward constrained control problem can be therefore expressed as follows:

$$
\min _{r(t)} t_{f}
$$

subject to:

$$
\begin{aligned}
& \frac{d \mathbf{x}(t)}{d t}=\mathbf{A} \mathbf{x}(t)+\mathbf{b} r(t) \quad 0 \leq t \leq t_{f} \\
& y(t)=\mathbf{c x}(t) \\
& \quad \mathbf{x}(0)=\mathbf{x}_{\mathbf{0}} \quad \mathbf{x}\left(t_{f}\right)=\mathbf{x}_{\mathbf{f}} \\
& u_{\min } \leq u(t) \leq u_{\max } \quad 0 \leq t \leq t_{f} \\
& y_{\min } \leq y(t) \leq y_{\max } \quad 0 \leq t \leq t_{f}
\end{aligned}
$$

where $(\mathbf{A}, \mathbf{b}, \mathbf{c})$ is minimal state-space realization of the closed-loop system dynamics

$$
H(s):=\frac{C(s) \tilde{P}(s)}{1+C(s) \tilde{P}(s)},
$$

$\mathbf{x}_{\mathbf{0}}$ and $\mathbf{x}_{\mathbf{f}}$ are the initial and final conditions associated to the output value $y_{0}$ and $y_{1}$ respectively, and $u_{m i n}$, $u_{\max }$ and $y_{\min }, y_{\max }$ are evidently the constraints for the control variable and the process variable respectively.

\subsection{Optimization based on Chebyshev polynomials}

The Chebyshev polynomials of the first kind are a set of orthogonal polynomials defined as the eigenfunctions of a singular Sturm-Liouville problem [12] and denoted $\bar{T}_{i}(\tau)$. They are normalized such that $\bar{T}_{i}(1)=1, i=0,1, \ldots$ and in their trigonometric form they are expressed as:

$$
\bar{T}_{i}(\tau)=\cos (i \cdot \arccos (\tau)) \quad \tau \in[-1,1] .
$$

They can be also defined by the recurrence relation:

$$
\begin{aligned}
& \bar{T}_{0}(\tau)=\quad 1 \\
& \bar{T}_{1}(\tau)=\quad \tau \\
& \bar{T}_{i+1}(\tau)=2 \tau \bar{T}_{i}(\tau)-\bar{T}_{i-1}(\tau) \quad i>1
\end{aligned}
$$

The methodology based on the Chebyshev polynomials consists in first applying the following time transformation:

$$
t=t(\tau)=\frac{t_{f}}{2}(1+\tau)
$$

which allows a change from the time domain $t \in\left[0, t_{f}\right]$ to the reference domain $\tau \in[-1,1]$. Then, the state of 
the system $\mathbf{x}$ and the command input $r$ can be approximated by a truncated Chebyshev series of order $h$, and, by applying the properties of the Chebyshev polynomials, the minimum-time control problem is converted into a constrained optimization problem that can be solved with standard optimization tools [11]. Eventually, the signal $r(t)=r(t(\tau))=\tilde{r}(\tau)$ that minimises the transition time is approximated by

$$
\tilde{r}_{h}(\tau)=\sum_{i=0}^{h} \beta_{i} \bar{T}_{i}(\tau)
$$

where $\boldsymbol{\beta}:=\left(\beta_{0}, \beta_{1}, \ldots, \beta_{h}\right)$ are coefficients found by the optimization procedure. Note that this signal can be expressed simply in the time domain variable $t$ by inverting formula (12), namely by applying the expression

$$
\tau=\tau(t)=\frac{2 t}{t_{f}}-1
$$

Indeed, the determined command function $r_{h}(t)=\tilde{r}_{h}(\tau)$ has to be applied in the time interval $t \in\left[0, t_{f}\right]$, after which the steady-state value $1 / H(0)$ has to be selected. Summarising, the command signal that has to be applied to the closed-loop system in order to attain the minimumtime process variable transition is:

$$
r(t)= \begin{cases}r_{h}(t) & \text { for } 0 \leq t \leq t_{f} \\ 1 / H(0) & \text { for } t>t_{f}\end{cases}
$$

\section{Determination of filter transfer function}

The purpose of the method described in this paper is to find a transfer function of a filter $F$ such that its step response approximates the determined command signal $r_{h}(t)$, in order to implement the devised technique by means of a standard two degree-of-freedom controller (see Fig.2). It is worth stressing at this point that $F(s)$ cannot be found by simply applying the inverse Laplace transform operator to $r_{h}(t)$ because this would yield an unstable transfer function with $h$ poles at the origin of the complex plane.

\subsection{Expression of the step response with truncated Chebyshev series}

For the purpose of finding a (low-order) asymptotically stable transfer function, it is convenient to consider again the reference domain $\tau \in[-1,1]$ and to rewrite the command function $\tilde{r}_{h}(\tau)$ in monomial form as follows:

$$
\tilde{r}_{h}(\tau)=\left[\xi_{0}, \xi_{1}, \ldots, \xi_{h}\right]\left[\begin{array}{c}
\tau^{0} \\
\tau^{1} \\
\vdots \\
\tau^{h}
\end{array}\right] .
$$

The vector

$$
\Xi:=\left[\xi_{0}, \xi_{1}, \ldots, \xi_{h}\right]
$$

can be expressed as

$$
\Xi=\left(\boldsymbol{\Gamma} \boldsymbol{\beta}^{T}\right)^{T}
$$

where

$$
\boldsymbol{\Gamma}=\left[\begin{array}{ccccc}
1 & 0 & \gamma_{1,3} & \cdots & \gamma_{1, h+1} \\
0 & 1 & \gamma_{2,3} & \cdots & \gamma_{2, h+1} \\
0 & 0 & \gamma_{3,3} & \cdots & \gamma_{3, h+1} \\
\vdots & \vdots & \vdots & \ddots & \vdots \\
0 & 0 & \gamma_{h+1,3} & \cdots & \gamma_{h+1, h+1}
\end{array}\right]
$$

and where each column $\gamma_{i}=$ $\left[\gamma_{1, i}, \gamma_{2, i}, \cdots, \gamma_{h+1, i},\right]^{T} \quad(i=1, \cdots, h+1)$ can be recursively evaluated by means of the relation

$$
\gamma_{i}=2 \bar{\gamma}_{i}-\gamma_{i-2}
$$

with

$\bar{\gamma}_{i}=\left[0, \gamma_{1, i-1}, \gamma_{2, i-1}, \cdots, \gamma_{h, i-1}\right]^{T} \quad i=3, \cdots, h+1$.

Then, we consider a filter transfer function which contains only real poles and zeros, namely,

$$
F(s)=k \frac{\prod_{j=1}^{m}\left(s-z_{j}\right)}{\prod_{j=1}^{n}\left(s-p_{j}\right)}, \quad n \geq m .
$$

Since all poles and zeros are real, the Laplace transform $R_{s}(s)$ of the response $r_{s}(t)$ of the filter $F(s)$ to a step signal of amplitude $y_{1}=1$, after having applied a partial fraction expansion, can be written as

$$
R_{s}(s)=\frac{1}{s} F(s)=\frac{a_{0}}{s}+\sum_{j=1}^{n} \frac{a_{j}}{s-p_{j}} .
$$

where the vector of coefficients $\mathbf{a}=\left[a_{0}, a_{1}, \cdots, a_{n}\right]$, which depends on the filter parameters

$$
\mathbf{v}=\left(k, p_{1} \ldots p_{m}, z_{1} \ldots z_{n}\right),
$$

can be easily computed by solving a linear system.

The inverse Laplace transform of (23) can be computed as

$$
\begin{aligned}
r_{s}(t) & =\mathcal{L}^{-1}\left\{\frac{a_{0}}{s}+\sum_{j=1}^{n} \frac{a_{j}}{s-p_{j}}\right\} \\
& =a_{0}+\sum_{j=1}^{n} a_{j} e^{p_{j} t}
\end{aligned}
$$

At this point, it is convenient to rewrite the step response (25) into the reference domain by applying (12) and this yields

$$
\begin{aligned}
r_{s}(t)=r_{s}(t(\tau))=\tilde{r}_{s}(\tau) & =a_{0}+\sum_{j=1}^{n} a_{j} e^{p_{j}\left(\frac{t_{f}}{2}(1+\tau)\right)} \\
& =a_{0}+\sum_{j=1}^{n} b_{j} e^{p_{j} \frac{t_{f}}{2} \tau}
\end{aligned}
$$

where

$$
b_{j}=a_{j} e^{p_{j} \frac{t_{f}}{2}}, \quad j=1, \ldots, n .
$$

By approximating the exponential terms of (26) by means of truncated Chebyshev series we obtain:

$$
\tilde{r}_{s}(\tau) \simeq \tilde{r}_{s, h}(\tau)=a_{0}+\sum_{j=1}^{n} b_{j} \sum_{i=0}^{h} \psi_{j i} \bar{T}_{i}(\tau)
$$


where the coefficients $\boldsymbol{\psi}_{j}=\left(\psi_{j 1}, \psi_{j 2}, \cdots, \psi_{j h}\right)$ are the coefficients of the $h$-degree Chebyshev projection polynomials which fits the exponential response generated by pole $j$. Chebyshev projection is performed as follows:

$$
\psi_{j k}=\frac{2}{\pi c_{k}} \sum_{i=0}^{h} e^{p_{j} \frac{t_{f}}{2} \tau_{i}} \bar{T}_{k}\left(\tau_{i}\right) w_{i}
$$

where

$$
c_{k}= \begin{cases}2 & k=0, h \\ 1 & k=1, \ldots, h-1\end{cases}
$$

and $\tau_{i}$ and $w_{i}$ are the Chebyshev Gauss-Lobatto nodes and weights, respectively, i.e. [12]:

$$
\begin{gathered}
\tau_{i}=\cos \frac{\pi i}{h}, \quad i=0, \ldots, h \\
w_{i}= \begin{cases}\frac{\pi}{2 h} & i=0, h \\
\frac{\pi}{h} & i=1, \ldots, h-1\end{cases}
\end{gathered}
$$

By applying a similar reasoning as in (16), the step response (28) can be written in monomial form as

$$
\begin{aligned}
\tilde{r}_{s, h}(\tau) & =a_{0}+\sum_{j=1}^{n} b_{j}\left(\boldsymbol{\Gamma} \psi_{j i}^{T}\right)^{T} \tau^{j} \\
& =\left[\omega_{0}+a_{0}, \omega_{1}, \cdots, \omega_{h}\right]\left[\begin{array}{c}
\tau^{0} \\
\tau^{1} \\
\vdots \\
\tau^{h}
\end{array}\right]
\end{aligned}
$$

where the coefficients $\left(\omega_{0}, \omega_{1}, \cdots, \omega_{h}\right)$ can be computed as

$$
\omega_{i}=\left(\sum_{j=1}^{n} b_{j} \psi_{i j}\right) \boldsymbol{\Gamma}^{T}, \quad i=1, \ldots, h,
$$

and they depend on $\mathbf{v}$.

\subsection{Optimization}

The polynomial $\tilde{r}_{s, h}(\tau)$ depends on the choice of poles, zeros and of the gain $k$ of the filter (22), i.e., $\tilde{r}_{s, h}(\tau)=$ $\tilde{r}_{s, h}(\tau ; \mathbf{v})$. An optimization algorithm has been devised in order to find the vector $\mathbf{v}$ (see (24)) such as $\tilde{r}_{s, h}(\tau ; \mathbf{v})$ is the best approximation of $\tilde{r}_{h}(\tau)$ with respect to a suitable norm. Formally, the optimization problem can be expressed as follows:

$$
\min _{\mathbf{v} \in \mathbb{R}^{n+m+1}}\left\|\tilde{r}_{h}-\tilde{r}_{s, h}\right\|
$$

We set

$$
\Omega=\left[\begin{array}{c}
\omega_{0}+a_{0} \\
\omega_{1} \\
\vdots \\
\omega_{h}
\end{array}\right] \in \mathbb{R}^{h}
$$

and

$$
\|\alpha\|_{w}:=\left(\sum_{j=0}^{h} \frac{\alpha_{j}^{2}}{j !}\right)^{\frac{1}{2}} \quad \forall \alpha \in \mathbb{R}^{h}
$$

A viable way to solve (35) is to consider the following performance index $J(\mathbf{v})$ :

$$
\begin{aligned}
J(\mathbf{v}) & =\|\Xi-\Omega\|_{w}^{2} \\
& =\left(\xi_{0}-\left(\omega_{0}+a_{0}\right)\right)^{2}+\sum_{j=1}^{h} \frac{\left(\xi_{j}-\omega_{j}\right)^{2}}{j !}
\end{aligned}
$$

where the factorial used in the denominator penalises high-order coefficients which can be very different (causing high values of $J(\mathbf{v})$ ) but they have a negligible effect on the shape of polynomial $\tilde{r}_{s, h}(\tau ; \mathbf{v})$.

Eventually, the optimization problem can be posed as:

$$
\min _{\mathbf{v} \in \mathbb{R}^{n+m+1}} J(\mathbf{v})
$$

subject to the inequality constraints

$$
\left[\begin{array}{c}
-\infty \\
-\infty \\
\vdots \\
-\infty \\
-\infty \\
\vdots \\
-\infty
\end{array}\right]<\left[\begin{array}{c}
k \\
p_{1} \\
\vdots \\
p_{m} \\
z_{1} \\
\vdots \\
z_{n}
\end{array}\right]<\left[\begin{array}{c}
+\infty \\
0 \\
\vdots \\
0 \\
+\infty \\
\vdots \\
+\infty
\end{array}\right]
$$

and to the equality constraint

$$
k \frac{\prod_{j=1}^{m} z_{j}}{\prod_{j=1}^{n} p_{j}}-\mu=0
$$

where $\mu$ is the gain of the filter which corresponds to the value of the polynomial $r_{h}(\tau)$ evaluated for $\tau=1$.

Optimization problem (39)-(41) can be solved using a sequential quadratic programming (SPQ) method, such as the one implemented in the function " $f m i n c o n$ " of Matlab [8]. Initial conditions are selected so that one pole is equal to $-5 / t_{f}$ (namely, it corresponds to a first-order system whose settling time is $t_{f}$ ) and then the other poles are slower. The zeros are selected so that one (negative) zero is closer to the imaginary axis than the poles (so that an overshoot is allowed) and another zero is positive (to address the presence of an apparent dead time in the response). Note that other (global) optimization methods, such as genetic algorithms, could be also effectively applied.

Remark 1. It is evident that the devised method can be employed with any polynomial signal, namely, in a context which is different than its application to a PID feedback control loop. Here we address this specific case because of its relevance in the industrial process control context.

\section{Practical considerations}

While practical considerations related to the Chebyshev optimization are addressed in [11], here we discuss the technique for the determination of the feedforward filter transfer function. The effectiveness of the proposed 


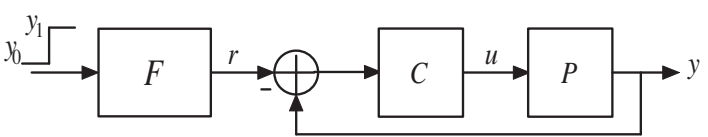

Figure 2. Standard two degree-of-freedom control scheme.

methodology depends on the shape of the polynomial signal $r_{h}(\tau)$ to be approximated. Indeed, the more regular and smooth is the signal, the higher will be the accuracy of the approximation provided by the (low-order) filter $F(s)$ determined by means of the optimization procedure described in the previous section. In fact the solution of the time-optimal control problem represents a generalized bang-bang, namely, the time-optimal control is characterized by the fact that either the manipulated input or the controlled process output saturates during the transient [10]. Thus, in case of a sluggish tuning of the PID controller, the command signal will assume bigger values trying to set the control and controlled variables on their limit values. On the contrary, an aggressive tuning of the controller implies that a smooth command signal, which is easier to approximate as a step response, results. In any case, as already mentioned, the standard design strategy for a two degree-of-freedom control scheme is to tune the feedback controller aggressively in order to obtain a better performance in the load disturbance rejection task and to recover the set-point tracking performance by smoothing the set-point step signal. Thus, the overall methodology is well suited for a two degree-of-freedom control scheme, providing a minimum-time response by taking into account process input and output constraints.

Further, it is worth stressing that the higher is the number of poles $n$ and zeros $m$ of $F(s)$, the higher is the accuracy of the methodology, and the higher is the order $h$ of the polynomial $r_{h}(\tau)$, the better will be the approximation since the order $h$ affects the number of points where the performance index (38) is evaluated. On the other hand, increasing $n, m$, and $h$ increases the complexity of the optimization problem and of the implementation of the filter.

\section{Illustrative Results}

\subsection{Example 1}

With the aim of showing the effectiveness of the proposed method we consider the following FOPDT system

$$
P(s)=\frac{1}{5 s+1} e^{-s}
$$

A process output transition from $y_{0}=0$ to $y_{1}=1$ is required. The constraints for the control variable are selected as $u_{\min }=0$ and $u_{\max }=1.8$, while those on the process variable are selected as $y_{\min }=-0.01$ and $y_{\max }=1.01$.

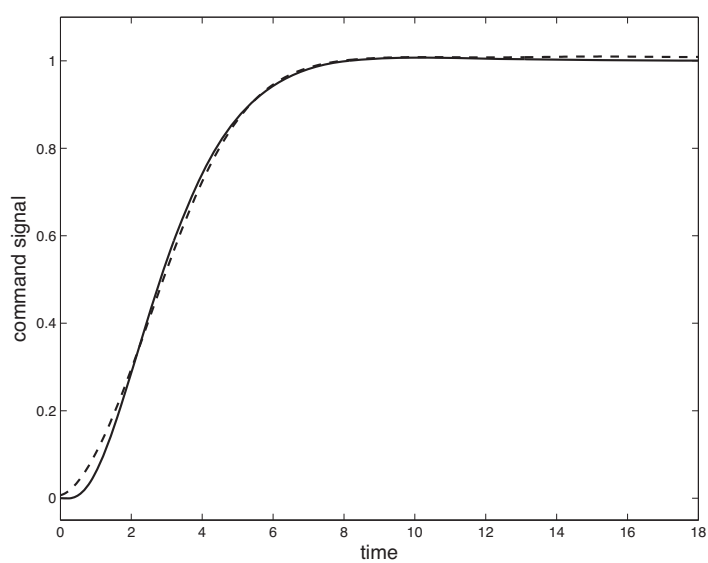

Figure 3. Minimum-time polynomial trajectory (dashed-line) and step response of the fourth-order filter with two zeros (solid-line) (43).

The parameters chosen for the PID controller (3) are $K_{p}=6, T_{i}=5, T_{d}=0.2, T_{f}=0.04$. This is an aggressive tuning (the corresponding phase margin is 32.8 degrees) which results in the (smooth) polynomial trajectory plotted as a dashed-line in Fig. 3 (note that $h=15$ has been selected). By selecting a filter structure with two zeros $(m=2)$ and four poles $(n=4)$, the filter transfer function $F(s)$ which results by applying optimization procedure is:

$$
F(s)=\frac{-0.097(s+0.38)(s-10.1)}{(s+0.51)(s+0.59)(s+0.79)(s+1.55)}
$$

The resulting process and control variables are plotted in Fig.4, where the dashed line refers to the use of $r_{h}(t)$ and the solid line to the use of $r_{s, h}(t)$.

In order to understand better the relationship between the effectiveness of the approximation and the tuning of the PID controller, in Fig.5 we show, for system (42), the approximation error $\|e\|_{\infty}=\left\|\tilde{r}_{h}-\tilde{r}_{s}\right\|_{\infty}=$ $\sup _{\tau \in[-1,1]}\left\|\tilde{r}_{h}(\tau)-\tilde{r}_{s}(\tau)\right\|$ for different PID parameters which yields different phase margins $\phi_{m}$. It appears that the approximation error, as mentioned in Section 4, decreases when the phase margin decreases.

\subsection{Example 2}

As a second example we consider the system [11]

$$
P(s)=\frac{1}{(s+1)^{8}}
$$

for which a FOPDT model has been derived, by applying the area method [2], as

$$
P(s)=\frac{1}{3 s+1} e^{-5 s}
$$



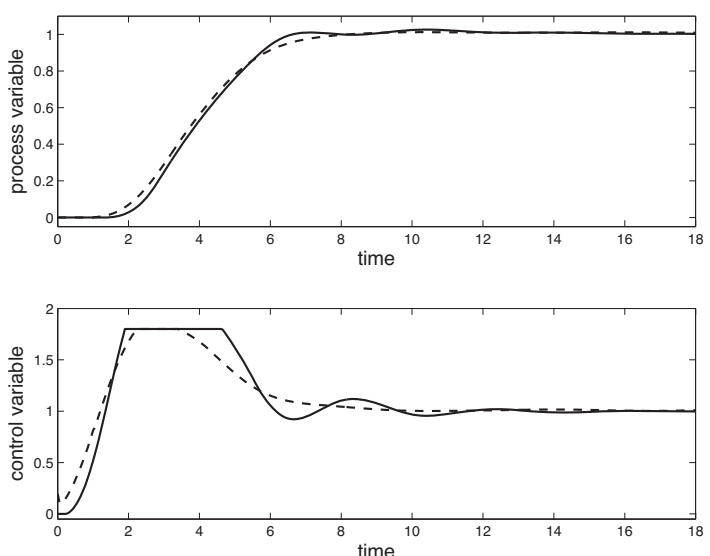

Figure 4. Process and control variables resulting by applying the minimum-time polynomial trajectory (dashed-line) and by the step response of the fourth order filter with two zeros (43) (solid-line) to the closed-loop system.

Again, a process output transition from $y_{0}=0$ to $y_{1}=1$ is required. The constraints for the control variable are selected as $u_{\min }=0$ and $u_{\max }=2, y_{\min }=-0.01$ and $y_{\max }=1.01$.

By selecting the PID parameters as $K_{p}=0.7, T_{i}=10$, $T_{d}=0.1, T_{f}=0.1$, the Chebyshev optimization $(h=$ 15) yields the polynomial trajectory plotted as a dashedline in Fig.6. By selecting again a filter structure with two zeros $(m=2)$ and four poles $(n=4)$, the filter transfer function $F(s)$ which results by applying optimization procedure is:

$$
F(s)=\frac{-0.064(s+0.055)(s-1.02)}{(s+0.31)(s+0.30)(s+0.29)(s+0.13)}
$$

The resulting process and control variables are plotted in Fig.7, where, as before, the dashed line refers to the use of $r_{h}(t)$ and the solid line to the use of $r_{s, h}(t)$.

\section{Conclusions}

In this paper we have addressed the problem of implementing a two degree-of-freedom control scheme which provides a minimum-time rest-to-rest transition subject to constraints on the process and control variables. We have proposed a technique for the design of a stable filter whose step response approximates a polynomial command function which yields the optimal transition. Practical issues have been addressed and therefore the overall method appears to be suitable to implement in industrial settings by means of Distributed Control Systems.

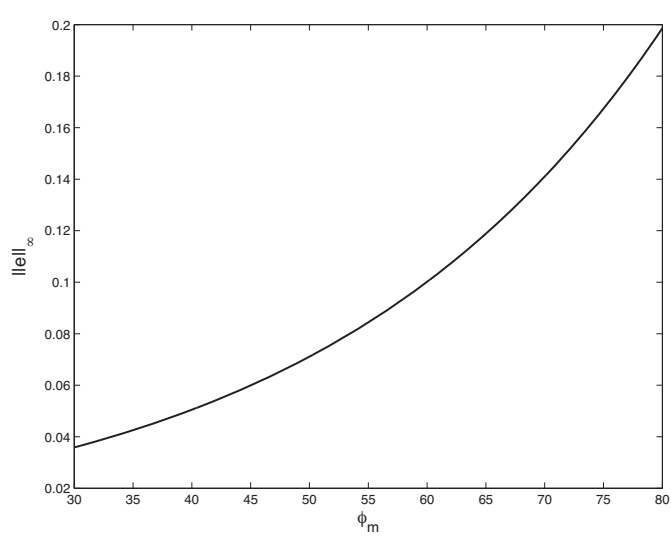

Figure 5. Behavior of the error $\|e\|_{\infty}$ depending on the phase margin $\phi_{m}$.

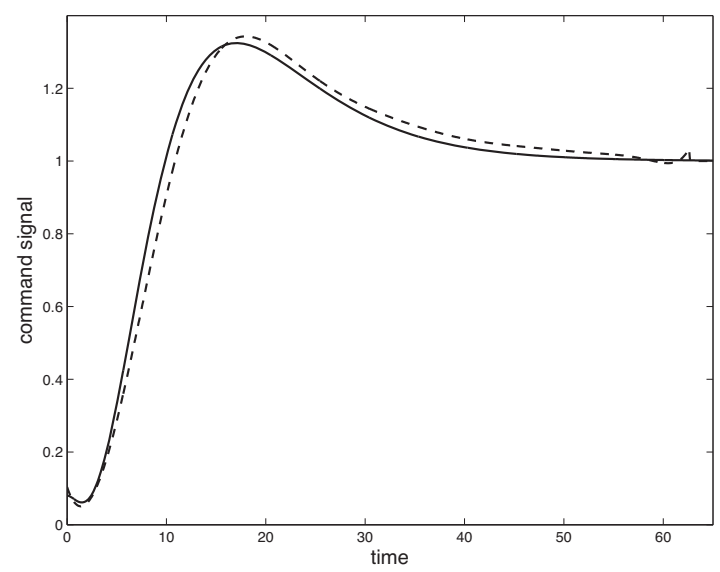

Figure 6. Minimum-time polynomial trajectory (dashed-line) and step response of the filter (46).

\section{References}

[1] M. Araki. Two degree-of-freedom PID controller. Systems, Control, and Information, 42:18-25, 1988.

[2] K. J. Åström and T. Hägglund. Advanced PID Control. ISA Press, Research Triangle Park, USA, 2006.

[3] K. Glover. All optimal Hankel norm approximation of linear multivariable systems, and their $l_{\infty}$ error bounds. International Journal of Control, 39:1145-1193, 1984.

[4] H. Jaddu and E. Shimemura. Computation of optimal control trajectories using Chebyshev polynomials: parametrization, and quadratic programming. Optimal Control Applications and Methods, 20:21-42, 1999.

[5] A. Leva and L. Bascetta. Designing the feedforward path of 2-d.o.f. industrial controllers for optimal tracking. Control Engineering Practice, 15:909-921, 2007.

[6] A. Leva and L. Bascetta. FIR based causal design of 2d.o.f. controllers for optimal set-point tracking. Journal of Process Control, 18:465-478, 2008. 

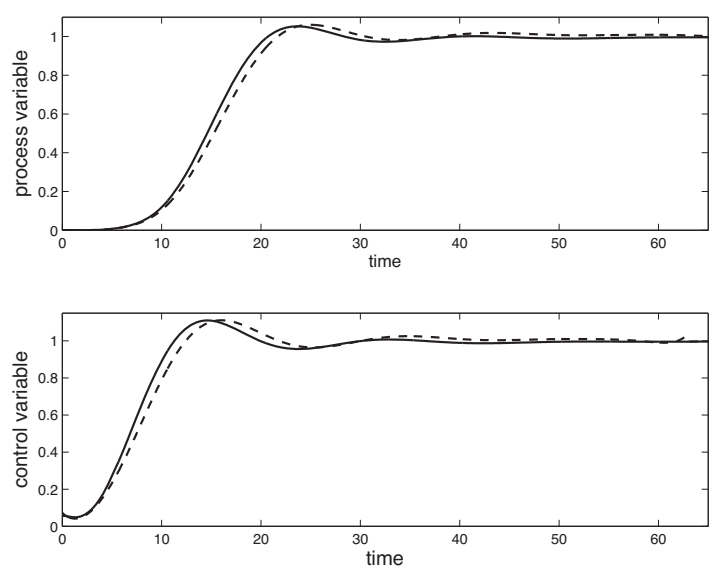

Figure 7. Process and control variables resulting by applying the minimum-time polynomial trajectory (dashed-line) and by the step response of the filter (46) (solid-line) to the closed-loop system.

[7] A. Leva, C. Cox, and A. Ruano. Hands-on PID autotuning: a guide to better utilisation. IFAC Professional Brief, 2001.

[8] Matlab. Optimization toolbox user's guide. The Mathworks, Natick (USA), 2006.

[9] A. O'Dwyer. Handbook of PI and PID Tuning Rules. Imperial College Press, 2006.

[10] A. Piazzi and L. Consolini. Generalized bang-bang control for feedforward constrained linear system. In Proceedings IEEE International Conference on Decision and Control, pages 893-898, 2006.

[11] S. Piccagli and A. Visioli. Minimum-time feedforward plus PID control using a Chebyshev technique. In Proceedings IEEE International Conference on Decision and Control, pages 1795-1800, 2007.

[12] A. Quarteroni and A. Valli. Numerical Approximation of Partial Differential Equations. Springer-Verlag, Berlin (D), 1997.

[13] M. G. Safonov and R. Y. Chiang. A Schur method for balanced model reduction. IEEE Transactions on Automatic Control, AC-2:729-733, 1989.

[14] A. Visioli. Practical PID Control. Springer, London(UK) 2006.

[15] J. Vlassenbroeck. A Chebyshev polynomial method for optimal control with state constraints. Automatica, 24(4):499-506, 1988 